\title{
Análisis de la composición química principal de materiales pétreos empleados en obras monumentales
}

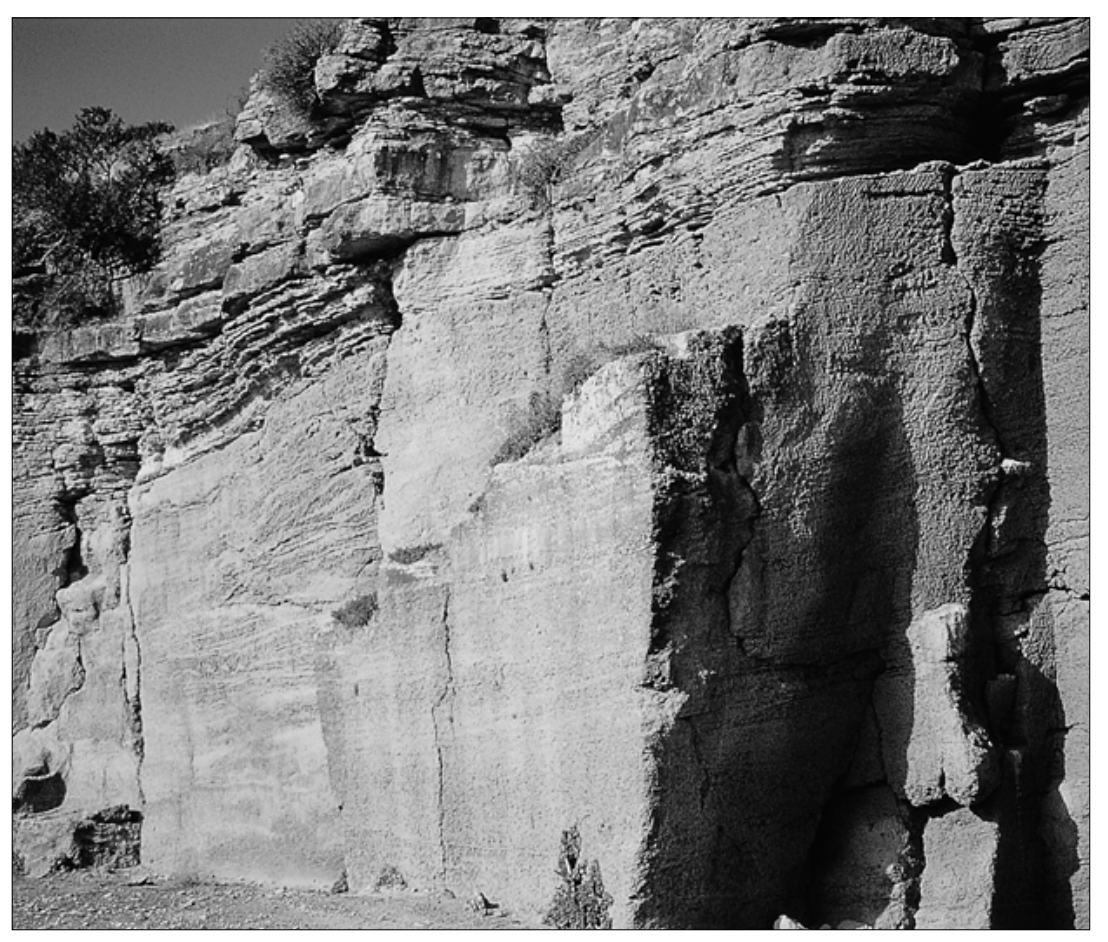

Miguel Ángel Bello Departamento de Química Analítica

Universidad de Sevilla

Antonio Martín Departamento de Química Edafología Universidad de Navarra

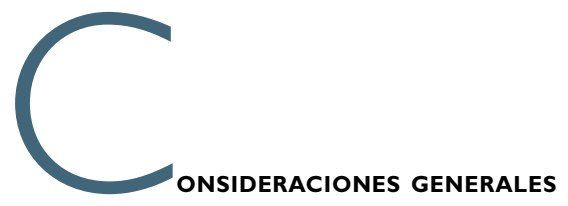

La composición química de los materiales pétreos, de sus productos de alteración o de materiales relacionados con la conservación de obras monumentales, es, en la mayor parte de los casos, un dato de gran interés realizar para una correcta caracterización de los mismos. En general, el análisis de componentes mayoritarios de tipo inorgánico se realiza mediante métodos químicos, empleándose métodos instrumentales para los componentes minoritarios y traza de tipo inorgánico, así como para los compuestos orgánicos.

La aplicación de ciertas técnicas fisicoquímicas también permite obtener datos morfológicos y de composición 
química puntual, de gran interés, por ejemplo, en el seguimiento de experiencias de alteración acelerada.

La composición química de los materiales pétreos puede proporcionar una información esencial en muy diversos campos de actuación relacionados con la alteración y conservación de materiales pétreos:

- Variación de la composición química de muestras alteradas e inalteradas, y en aquéllas, con la profundidad.

- Comparación entre muestras alteradas para valorar el tipo e intensidad de la alteración.

- Conocer cuales son los factores y mecanismos de alteración implicados.

- Localización e identificación de las canteras de procedencia de los materiales empleados en el monumento.

- Datación de ciertas estructuras y partes del monumento en función de los tipos de materiales empleados.

- Investigaciones relacionadas con productos de conservación.

Dada la amplitud del tema, en este artículo nos centraremos en el análisis de los componentes principales, mayoritarios y minoritarios, de los materiales pétreos, la denominada composición porcentual, quedando para otro u otros artículos aspectos tales como el análisis de componentes traza, de sales solubles, de compuestos orgánicos y los aspectos relacionados con la toma de muestra, de primordial importancia para garantizar la representatividad de las posteriores determinaciones analíticas.

\section{ANÁLISIS DE LOS COMPONENTES PRINCIPALES}

El análisis de los componentes principales de un material pétreo consiste en la determinación de diez parámetros que se expresan como porcentajes en peso de la muestra original seca a $105^{\circ} \mathrm{C}$ :

Pérdida por calcinación, que corresponde, fundamentalmente, al dióxido de carbono que se desprende como consecuencia de la descomposición de los carbonatos; también se pierde el agua de hidratación de aquellas sales que la pierdan por encima de $105^{\circ} \mathrm{C}$.

$$
\begin{aligned}
& -\mathrm{SiO}_{2} \\
& -\mathrm{Al}_{2} \mathrm{O}_{3} \\
& -\mathrm{Fe}_{2} \mathrm{O}_{3} \\
& -\mathrm{TiO}_{2} \\
& -\mathrm{CaO} \\
& -\mathrm{MgO} \\
& -\mathrm{Na}_{2} \mathrm{O} \\
& -\mathrm{K}_{2} \mathrm{O} \\
& -\mathrm{SO}_{3}
\end{aligned}
$$

La suma de los porcentajes obtenidos debe quedar, para materiales típicos, entre el 99.50 y el $\mathbf{1 0 0 . 5 0 \% ,}$ asegurándose de esta forma el correcto análisis del material.

Se parte de material seco a $105^{\circ} \mathrm{C}$ y molido hasta un nivel suficiente que permita un fácil ataque, y cuyas manipulaciones no hayan provocado contaminaciones accidentales de la muestra.

Los reactivos empleados deben ser de calidad para análisis, y en todo caso se debe garantizar que su empleo no provoque alteraciones en la composición de la muestra analizada que afecten al resultado del análisis.

En lo que respecta al material volumétrico a usar, este debe ser de una adecuada calidad, y los instrumentos de medida deben estar perfectamente calibrados, empleándose para las pesadas una balanza analítica.

Por último, hacer notar que en el caso de que se proponga más de un procedimiento de determinación para algún parámetro, la elección vendrá dada por la cantidad presente; así, para pequeñas cantidades de magnesio es preferible la determinación mediante espectrofotometría de absorción atómica, y si el contenido de sulfatos en la muestra es bajo, se prefiere su determinación turbidimétrica.

\section{DETERMINACIÓN DE LA PÉRDIDA POR CALCINACIÓN}

\author{
Aparatos \\ - Horno mufla $\left(950^{\circ} \mathrm{C}\right)$ \\ - Balanza analítica
}

\section{Procedimiento}

I. Tarar a $950^{\circ} \mathrm{C}$ una navecilla de porcelana de fondo bajo hasta peso constante.

2. Pesar en la navecilla ya tarada 0.7 gramos de muestra finamente pulverizada y desecada a $105^{\circ} \mathrm{C}$.

3. Calcinar a $950^{\circ} \mathrm{C}$ durante dos horas, enfriar en desecador y pesar.

4. Repetir la operación hasta pesada constante.

5. Calcular la pérdida de peso que se ha producido y expresarla como porcentaje.

\section{DETERMINACIÓN DE SÍLICE}

\section{Reactivos y aparatos}

- Ácido clorhídrico concentrado

- Ácido nítrico concentrado

- Solución de gelatina al 2.5\% (p/v). Disolver gelatina pura (sin residuo de calcinación) en la cantidad ne- 
cesaria de agua, calentando en baño-maría. La solución es estable durante una semana.

- Solución de lavado. Añadir $2 \mathrm{ml}$ de $\mathrm{HCl}$ concentrado y $2 \mathrm{ml}$ de solución de gelatina al $2.5 \%$ a cada litro de solución de lavado.

- Mezcla de carbonato sódico-potásico y boráx: Mezclar íntimamente $34 \mathrm{~g}$ de $\mathrm{NaKCO}_{3}$ y $23 \mathrm{~g}$ de $\mathrm{Na}_{2} \mathrm{~B}_{4} \mathrm{O}_{7} 10 \mathrm{H}_{2} \mathrm{O}$.

- Horno mufla

\section{Procedimiento}

I. Pesar $0.7 \mathrm{~g}$ de muestra, finamente pulverizada en mortero de ágata y desecada a $105^{\circ} \mathrm{C}$, en un crisol de platino con tapadera.

2. Mezclar íntimamente con 3.I g de mezcla $\mathrm{NaKCO}_{3-}$ bórax.

3. Fundir a $950^{\circ} \mathrm{C}$.

4. Disolver el fundido, introduciendo crisol y tapadera en un vaso de $400 \mathrm{ml}$ y agregando unos $150 \mathrm{ml}$ de agua destilada y $50 \mathrm{ml}$ de $\mathrm{HCl} \mathrm{l}: \mathrm{l}$.

5. Sacar el crisol y tapadera lavándolas con agua destilada.

6. Evaporar a sequedad en baño de arena.

7. Añadir $17.5 \mathrm{ml}$ de $\mathrm{HCl}$ concentrado haciéndolo resbalar sobre las paredes del vaso a fin de arrastrar las sales al fondo.

8. Hervir durante unos 6 minutos, cubriendo el vaso con un vidrio de reloj.

9. Agregar, a continuación, I ml de $\mathrm{HCl}$ concentrado y $10 \mathrm{ml}$ de agua.

10. Calentar a $70^{\circ} \mathrm{C}\left( \pm 5^{\circ} \mathrm{C}\right)$.

II. Agregar, gota a gota y agitando, $6.5 \mathrm{ml}$ de la solución de gelatina previamente calentada a igual temperatura.

12. Dejar en reposo durante unos 25 minutos.

13. Filtrar sobre papel Albet 242 o similar, recogiendo el filtrado sobre un matraz aforado de $250 \mathrm{ml}$.

14. Lavar con unos $30 \mathrm{ml}$ de solución de lavado caliente recogiéndolos sobre el mismo matraz.

15. Enrasar el matraz, una vez frío, con agua destilada, y esta solución se reservará y se rotula como solución $A$.

16. Trasladar el papel con el precipitado a un crisol previamente tarado y secar en la estufa a $105^{\circ} \mathrm{C}$.

17. Calcinar a $1000^{\circ} \mathrm{C}$, con las precauciones normales.

18. Enfriar en un desecador y pesar.

19. Calcular el contenido de $\mathrm{SiO}_{2}$ expresado como porcentaje en la muestra original.

\section{PREPARACIÓN DE LA SOLUCIÓN B} (SEPARACIÓN DE SESQUIÓXIDOS)

\section{Reactivos}

- Acido nítrico concentrado

- Hidróxido amónico $(1: 1)$

- Solución de nitrato amónico al 2\% (p/v)

\section{Procedimiento}

I. Tomar $100 \mathrm{ml}$ de la solución anterior (solución A) en un vaso de $250 \mathrm{ml}$.

\section{Agregar unas gotas de $\mathrm{HNO}_{3}$ concentrado.}

3. Calentar a ebullición y añadir $\mathrm{NH}_{4} \mathrm{OH}(\mathrm{I}: \mathrm{I})$, gota a gota y agitando, hasta débil olor permanente a amoníaco.

4. Hervir durante I-2 minutos.

5. Filtrar por papel de filtro de tamaño de poro medio (Albet 238 o 240), recogiendo el filtrado en un matraz aforado de $250 \mathrm{ml}$.

6. Lavar el precipitado con solución caliente de $\mathrm{NH}_{4} \mathrm{NO}_{3}$ al $2 \%$ hasta total eliminación de cloruros y añadir las aguas de lavado al matraz aforado.

7. Disolver el precipitado con $\mathrm{HCl}$ diluido caliente, lavando el papel con agua caliente.

8. Reprecipitar los hidróxidos, filtrar y lavar como se expuso anteriormente llevando los lavados al matraz aforado de $250 \mathrm{ml}$.

9. Enrasar con agua destilada y reservar para la determinación de calcio y magnesio. (Solución B).

\section{DETERMINACIÓN DE CALCIO}

\section{Reactivos}

- Solución de hidróxido sódico 2 M

- Indicador de ácido calconcarboxílico. Solución al $0.4 \%$ en metanol o trituración con sulfato sódico anhidro al $1 \%$

- Indicador de calceína. Pulverizar en mortero de ágata y mezclar íntimamente 0.004 g de calceína y I g de cloruro sódico. El indicador así preparado tiene una duración de unos 10 días

- Solución AEDT 0.01 M. Disolver $3.7225 \mathrm{~g}$ de la sal en agua destilada y llevar a I litro en matraz aforado. Valorar con una solución patrón de calcio.

- Solución patrón de calcio: Pasar $1.000 \mathrm{~g}$ de $\mathrm{CaCO}_{3}$ de calidad reactivo análisis y previamente desecado a $105^{\circ} \mathrm{C}$, a un matraz aforado de I litro, con ayuda de un embudo. Lavar el polvo del embudo y el cuello del matraz con agua destilada. Añadir $250 \mathrm{ml}$ de $\mathrm{HCl} 0.1 \mathrm{~N}$ y agitar hasta disolución total. Añadir $40 \mathrm{ml}$ de $\mathrm{NaOH}$ 0.I N. Enfriar a temperatura ambiente y enrasar con agua destilada. 
- Cianuro potásico

- Clorhidrato de hidroxilamina

\section{Procedimiento}

I. Pipetear, en un matraz erlenmeyer de $250 \mathrm{ml}$, una alícuota de la solución procedente de precipitar los hidróxidos (solución B). Dicha alícuota no debe contener más de $50 \mathrm{mg}$ de ión calcio e ión magnesio. Normalmente se emplearán $50 \mathrm{ml}$.

2. Hervir con dos lentejas de $\mathrm{KOH} \circ \mathrm{NaOH}$ para eliminar el exceso de amoníaco.

3. Enfriar y diluir a unos $50 \mathrm{ml}$.

4. Mezclar perfectamente con unos $10 \mathrm{ml}$ de solución de $\mathrm{NaOH}$, a fin de que precipite todo el magnesio y la solución tenga un $\mathrm{pH}$ no inferior a 12, comprobar con papel indicador.

5. Dejar reposar durante 3-5 minutos, agitando de vez en cuando.

6. Añadir un poco de KCN y de clorhidrato de hidroxilamina para evitar posibles interferencias de pequeñas cantidades de cobre, manganeso, cinc, cobalto o niquel.

7. Añadir 5-10 gotas de solución de indicador de ácido calconcarboxílico, 0,2-0,4 g de la mezcla sólida del mismo ó 0,log de indicador de calceína.

8. Valorar con solución de AEDT 0,0I M previamente contrastada, agitando vigorosamente (se aconseja emplear un agitador magnético) hasta que el color cambie de rojo vino a azul puro o verde fluorescente a azul-violeta y desaparezca la fluorescencia, según que se utilice como indicador ácido calconcarboxílico o calceína, respectivamente. En el caso de utilizar calceína, la adición de I-2 mg de azul de timol hace el viraje más perceptible.

9. Calcular el porcentaje de $\mathrm{CaO}$ en la muestra original teniendo en cuenta que I ml de AEDT 0,0 I M equivale a $0.56 \mathrm{mg}$ de $\mathrm{CaO}$.

10. En el caso de una concentración de magnesio relativamente elevada es aconsejable valorar el calcio por retroceso.

\section{DETERMINACIÓN DE MAGNESIO}

\section{DETERMINACIÓN VOLUMÉTRICA}

\section{Reactivos}

- Solución tampón. Disolver $54 \mathrm{~g}$ de $\mathrm{NH}_{4} \mathrm{Cl}$ en $300 \mathrm{ml}$ de agua destilada, añadir $444 \mathrm{ml}$ de $\mathrm{NH}_{4} \mathrm{OH} 12 \mathrm{~N}$ y enrasar a I litro con agua destilada.

- Indicador de negro de eriocromo T. Mezclar íntimamente en un mortero de ágata 3 partes de negro de eriocromo T y 997 partes de cloruro sódico.

- Solución de AEDT 0,05 M. Disolver 18.6127g de la sal en agua y enrasar a I litro en matraz aforado.

- Solución patrón de magnesio. Disolver $2.03 \mathrm{~g}$ de $\mathrm{MgCl}_{2} 6 \mathrm{H}_{2} \mathrm{O}$ de calidad reactivo análisis y desecado a 60C, en un pequeño volumen de agua y llevar a I litro en matraz aforado.

\section{Procedimiento}

I. Tomar una alícuota de $50 \mathrm{ml}$ de la solución procedente de precipitar los hidróxidos (solución B) en un matraz erlenmeyer de $250 \mathrm{ml}$. Dicha alícuota no debe contener más de $50 \mathrm{mg}$ de iones calcio y magnesio.

2. Añadir $10 \mathrm{ml}$ de solución tampón, a fin de llevar la solución a pH 10.

3. Diluir a unos $100 \mathrm{ml}$ con agua destilada.

4. Calentar a unos $40-50^{\circ} \mathrm{C}$.

5. Añadir una poco de KCN y de clorhidrato de hidroxilamina para evitar posibles interferencias de pequeñas cantidades de cobre, manganeso, cinc, cobalto o niquel.

6. Agregar unos $0.3 \mathrm{~g}$ de indicador.

7. Valorar con solución de AEDT 0.05 M mediante una microbureta.

8. Calcular el porcentaje de $\mathrm{MgO}$, teniendo en cuenta que I $\mathrm{ml}$ de AEDT $0.05 \mathrm{M}$ equivale a $2.016 \mathrm{mg}$ de $\mathrm{MgO}$ y que en esta valoración se determinan calcio y magnesio conjuntamente, por lo que se habrá de restar a la cantidad de AEDT consumida la requerida para la valoración del calcio, teniendo presente las correspondientes molaridades del AEDT.

\section{DETERMINACIÓN POR ABSORCIÓN ATÓMICA}

\section{Reactivos y aparatos}

- Espectrofotómetro de absorción atómica

- Solución patrón de magnesio

\section{Procedimiento}

I. Medir la absorción atómica a 202.6 nm con una llama aire-acetileno de la solución procedente de separar los sesquióxidos (solución B).

2. Construir una recta de calibrado medida en las mismas condiciones con soluciones conteniendo entre 2 y $10 \mathrm{mg} \mathrm{t}^{-1}$ de magnesio.

3. Expresar el contenido de magnesio como porcentaje de $\mathrm{MgO}$ en la muestra original.

\section{DETERMINACIÓN DE ALUMINIO E HIERRO}

Reactivos y aparatos

- Espectrofotómetro de absorción atómica

- Solución patrón de hierro

- Solución patrón de aluminio 


\section{Procedimiento}

I. Determinar el contenido de hierro en la solución A por medida de la absorción atómica a $280.0 \mathrm{~nm}$ con una llama aire-acetileno, y empleando como recta de calibrado soluciones de hierro conteniendo entre 0.1 y $5.0 \mathrm{mg} \mathrm{H}^{-1}$ de hierro.

2. Expresar la cantidad de hierro obtenido como porcentaje de $\mathrm{Fe}_{2} \mathrm{O}_{3}$ en la muestra original.

3. Determinar el contenido de aluminio en la solución A por medida de la absorción atómica a $309.3 \mathrm{~nm}$ con una llama nitroso-acetileno usando como recta de calibrado soluciones de aluminio conteniendo entre 2.0 y $100.0 \mathrm{mgl}-1$ de aluminio.

4. Expresar la cantidad de aluminio obtenido como porcentaje de $\mathrm{Al}_{2} \mathrm{O}_{3}$ en la muestra original.

\section{DETERMINACIÓN DE TITANIO}

\section{Reactivos y aparatos}

- Peróxido de hidrógeno (20 volúmenes)

- Mezcla sulfúrico/fosfórico. Mezclar 300 ml de agua, $200 \mathrm{ml}$ de ácido sulfúrico concentrado y $500 \mathrm{ml}$ de ácido fosfórico concentrado

- Solución patrón de titanio (40 mg t-1 de $\left.\mathrm{TiO}_{2}\right)$

- Espectrofotómetro visible

Procedimiento

I. Añadir alícuotas de $50 \mathrm{ml}$ de solución A sobre dos matraces aforados de $100 \mathrm{ml}$.

2. Añadir a cada uno $5 \mathrm{ml}$ de mezcla sulfúrico/fosfórico.

3. Agregar, únicamente a uno de los matraces, $10 \mathrm{ml}$ de peróxido de hidrógeno. Si se sospechase la presencia de vanadio debe añadirse $\mathrm{NaF}$ para evitar su interferencia.

4. Mezclar bien, mediante agitación.

5. Enrasar ambos matraces con agua destilada.

6. Medir la absorbancia, de la muestra que contiene peróxido de hidrógeno, a $410 \mathrm{~nm}$ frente a la que no lo contiene.

7. Operando de igual forma, preparar una recta de calibrado entre 2 y $10 \mathrm{mg} \mathrm{l}^{-1}$ de $\mathrm{TiO}_{2}$.

8. Usando la recta de calibrado, relacionar la lectura obtenida con $\mathrm{mg} \mathrm{l}^{-1}$ de $\mathrm{TiO}_{2}$ y expresar el resultado como porcentaje de $\mathrm{TiO}_{2}$.

\section{DETERMINACIÓN DE SODIO Y POTASIO}

\section{Reactivos y aparatos}

- Ácido clorhídrico concentrado

- Ácido nítrico concentrado
- Ácido fluorhídrico concentrado

- Solución patrón de cloruro sódico

- Solución patrón de cloruro potásico

- Espectrofotómetro de emisión de llama

\section{Procedimiento}

I. Pesar exactamente, en una cápsula de teflón, I g de muestra, finamente pulverizada en mortero de ágata y desecada a $105^{\circ} \mathrm{C}$.

2. Añadir lentamente $10 \mathrm{ml} \mathrm{de} \mathrm{HCl}$ concentrado, $4 \mathrm{ml}$ de $\mathrm{HNO}_{3}$ concentrado y $40 \mathrm{ml}$ de $\mathrm{HF}$ concentrado.

3. Llevar a sequedad en baño de arena.

4. Añadir $5 \mathrm{ml}$ de $\mathrm{HCl}$ y $0.5 \mathrm{ml}$ de $\mathrm{HNO}_{3}$ y llevar de nuevo a sequedad.

5. Repetir una vez más el tratamiento del apartado anterior.

6. Extraer el resíduo seco con $17.5 \mathrm{ml}$ de ácido clorhídrico concentrado y un poco de agua caliente y pasar el líquido a un matraz aforado de $250 \mathrm{ml}$.

7. Enrasar con agua destilada.

8. Medir la emisión atómica del sodio a $589.0 \mathrm{~nm}$ en una llama aire-acetileno.

9. Llevar el valor obtenido a la curva de calibración obtenida para soluciones conteniendo entre 4 y $40 \mathrm{mg}$ $1-1$ de sodio medidas en las mismas condiciones.

10. Expresar el resultado como porcentaje de $\mathrm{Na}_{2} \mathrm{O}$ en la muestra original.

II. Medir la emisión atómica del potasio a $766.5 \mathrm{~nm}$ en una llama aire-acetileno.

12. Llevar el valor obtenido a la curva de calibración obtenida para soluciones conteniendo entre 2 y $40 \mathrm{mg} \mathrm{I-1}^{-1}$ de potasio medidas en las mismas condiciones.

13. Expresar el resultado como porcentaje de $\mathrm{K}_{2} \mathrm{O}$ en la muestra original.

\section{DETERMINACIÓN DE SULFATOS}

\section{DETERMINACIÓN TURBIDIMÉTRICA}

\section{Reactivos y aparatos}

- Solución precipitante. Preparar una disolución con $20 \mathrm{~g}$ de acetato de bario, $75 \mathrm{ml}$ de ácido acético $10 \mathrm{~N}$ y $25 \mathrm{ml}$ de solución de goma arábiga al 5\% en agua. Una vez mezclados y disueltos se filtra. Esta solución es estable al menos durante un mes.

- Solución patrón de sulfato de $480 \mathrm{mg}^{-1}$

- Espectrofotómetro visible

\section{Procedimiento}

I. Tomar en matraces de $50 \mathrm{ml}$ una cantidad adecuada de disolución procedente de la eliminación de la sí- 
lice (solución A), de tal manera que quede dentro de la recta de calibrado que se haya construido.

2. Añadir I ml de solución precipitante y enrasar con agua destilada.

3. Agitar durante 2 minutos y dejar en reposo durante 10 minutos.

4. Medir en espectrofotómetro la absorbancia a 425 $\mathrm{nm}$, frente a un blanco preparado de igual forma pero sin sulfato, como máximo en los 5 minutos siguientes.

5. Los resultados se expresan como porcentaje de $\mathrm{SO}_{3}$ en la muestra original.

\section{DETERMINACIÓN GRAVIMÉTRICA}

\section{Reactivos}

- Solución de cloruro de bario al 10\%

- Solución de ácido clorhídrico 1:5

- Solución de nitrato de plata 0.I N

\section{Procedimiento}

I. Pesar entre 0.5 y I g de muestra finamente pulverizada y desecada a $105^{\circ} \mathrm{C}$.
2. Atacar con $50 \mathrm{ml}$ de $\mathrm{ClH} \mathrm{I:5} \mathrm{en} \mathrm{un} \mathrm{vaso} \mathrm{de} \mathrm{precipi-}$ tado llevando a ebullición.

3. Añadir a continuación $100 \mathrm{ml}$ de agua hirviente y mantener a ebullición durante otros 5 minutos.

4. Filtrar por papel Albet 240 o similar lavando el precipitado con agua caliente.

5. El filtrado obtenido se lleva a ebullición y se añaden, gota a gota, $20 \mathrm{ml}$ de solución caliente de cloruro de bario.

6. Dejar reposar el precipitado formado durante unos 5 minutos y comprobar que la precipitación ha sido completa.

7. Calentar de forma suave durante una hora y filtrar en caliente a través de filtro Albet 240 o similar.

8. Lavar el precipitado con pequeñas porciones de agua caliente hasta total eliminación de cloruros.

9. Colocar el filtro en un crisol de porcelana previamente tarado, secar en estufa y calcinar a $800^{\circ} \mathrm{C}$.

10. Enfriar en desecador y pesar.

II. Repetir el proceso de calcinación hasta pesada constante.

12. Expresar el peso de sulfato de bario obtenido como porcentaje de $\mathrm{SO}_{3}$ en la muestra original.

\section{Bibliografía recomendada}

BELLO, M. A. Caracterización y estado de alteración química de los materiales empleados en la construcción de la catedral de Sevilla. Tesis Doctoral. Universidad de Sevilla, 1988.

BERZOSA, l.; MARTÍN, A. Análisis rápido de materiales calizos y silíceos. Ensayos e Investigación, I.3, I.4. y I.5, 1966.

CHARLOT, G. Les méthodes de la chimie analytique. Analyse quantitative minérale. Ed. Masson et Cie., París, 1966.
DUPAS. M. L' analyse chimique des pierres calcaires et de leurs alteration dans les monuments. ICOMOS, Troisième Colloque sur l' Alteration des Pierres, Bruselas, I|-|4/X||/|981, 69-79.

MARTíN, A. Ensayos y experiencias de alteración en la conservación de obras de piedra de interés artístico. Editorial Centro de Estudios Ramón Areces. Madrid, 1990. 